\title{
Quantitative definitions of pain, CA19-9, and tumor size as high- risk features of resectable pancreatic cancer: a single-center retrospective cohort study
}

\author{
Dongni Xu ${ }^{1 \#}$, Jiangling Wang ${ }^{2 \#}$, Ting Liu ${ }^{1}$, Zhuoshan Huang ${ }^{3}$, Jianwei Luo ${ }^{1}$, Yuqing Chen ${ }^{1}$, Yanan Lu ${ }^{1}$ \\ ${ }^{1}$ Department of Anesthesiology, Sun Yat-sen Memorial Hospital, Sun Yat-sen University, Guangzhou, China; ${ }^{2}$ Department of Anesthesiology, Cancer \\ Hospital of Chinese Academy of Sciences, Hangzhou, China; ${ }^{3}$ Department of Stomatology, Sun Yat-sen Memorial Hospital, Sun Yat-sen University, \\ Guangzhou, China \\ Contributions: (I) Conception and design: D Xu, Y Lu, Y Chen; (II) Administrative support: Y Chen, J Wang; (III) Provision of study materials \\ or patients: J Wang, Y Lu; (IV) Collection and assembly of data: D Xu, T Liu, J Luo; (V) Data analysis and interpretation: D Xu, Z Huang; (VI) \\ Manuscript writing: All authors; (VII) Final approval of manuscript: All authors. \\ \#These authors contributed equally to this work and should be considered co-first authors. \\ Correspondence to: Yanan Lu, MD; Yuqing Chen, MD, PhD. Department of Anesthesiology, Sun Yat-sen Memorial Hospital, Sun Yat-sen University, \\ Guangzhou, China. Email: luynan@mail.sysu.edu.cn; chenyq97@mail.sysu.edu.cn.
}

Backgroundk Pancreatic ductal adenocarcinoma (PDAC) is one of malignant tumors with the worst prognosis. Surgery and adjuvant chemotherapy are the main treatments for resectable pancreatic cancer. For borderline resectable PDAC, neoadjuvant chemotherapy has been advised. For clearly resectable PDAC, neoadjuvant chemotherapy also might be considered for the patients with high-risk features, but with no precise quantitative criteria to define these features. So, this study aimed to re-evaluate the relationship between high-risk features and prognosis of clearly resectable pancreatic cancer, and to define the precise criteria for these high-risk features.

Methods: Data from 211 patients with clearly resectable pancreatic cancer were reviewed to assess the relationship between overall survival (OS) after surgery and high-risk features, and cut-off values were determined for high-risk features that were associated with poor prognosis of clearly resectable pancreatic cancer.

Results: Lymph node metastasis (LNM), neutrophil-to-lymphocyte ratio (NLR), platelet-to-lymphocyte ratio (PLR), and primary tumor size $\geq 6 \mathrm{~cm}$ were significant variables related to OS. CA19-9 $\geq 1,000 \mathrm{U} / \mathrm{mL}$ was statistically related to prognosis, as was CA19-9 $\geq 500 \mathrm{U} / \mathrm{mL}$ without obstructive jaundice. There was no significant relationship between abdominal and/or back pain and OS, but patients with moderate or severe pain accompanied by tumor size $\geq 4 \mathrm{~cm}$ or 10 times higher CA19-9 levels had worse prognosis.

Conclusions: For clearly resectable pancreatic cancer with R0 resection, the high-risk features were clarified. Abdominal and/or back pain may not be used as a prognostic indicator alone, though combined with CA19-9 or tumor size it may be more valuable for predicting prognosis.

Keywords: Resectable pancreatic cancer; carbohydrate antigen 19-9 (CA19-9); abdominal and/or back pain; primary tumor size; lymph node metastasis; prognosis; pancreatitis

Submitted Nov 23, 2020. Accepted for publication Feb 03, 2021.

doi: 10.21037 /gs-20-877

View this article at: http://dx.doi.org/10.21037/gs-20-877 


\section{Introduction}

Pancreatic ductal adenocarcinoma (PDAC) is one of the most aggressive malignant tumors with a poor prognosis, and is difficult to diagnose and treat. At present, for resectable pancreatic cancer, surgery and adjuvant chemotherapy are the main treatments (1-3). Since 1985, more and more evidence has confirmed the improved outcomes with adjuvant chemotherapy following resection of pancreatic cancer (2-4). Neoadjuvant chemotherapy has also been considered as effective for borderline resectable PDAC with the goal of improving overall survival (OS) $(5,6)$. Moreover, for clearly resectable PDAC with highrisk features, such as highly elevated CA 19-9, large primary tumors, large regional lymph nodes, and extreme pain, neoadjuvant chemotherapy may also be considered, as recommended by the National Comprehensive Cancer Network (NCCN) Guidelines Version 1.2019 (1). However, there are no precise quantitative criteria to define these high-risk features. Vague definitions such as "very highly", "large", and "extreme", have limited these guidelines in clinical practice. For example, a few studies concerning pain in clearly resectable PDAC showed "extreme pain" was actually rare in clinical practice, and $11.6 \%$ of patients with resectable diseases had severe pain which could be controlled by oral non-steroidal anti-inflammatory drugs (NSAIDs) (7). Although there were many reports about high-risk features for resectable PDAC, but most of them studied all resectable diseases (combine borderline resectable diseases and clearly resectable diseases). Rare reports separate studied the high-risk features for clearly resectable PDAC. Therefore, we aimed to review the data of patients who underwent pancreatic cancer surgery in recent years in order to re-evaluate the relationship between high-risk features and prognosis of clearly resectable pancreatic cancer, and obtain quantitative definitions of high-risk features.

We present the following article in accordance with the STROBE reporting checklist (available at http://dx.doi. org/10.21037/gs-20-877).

\section{Methods}

\section{Patients}

This study reviewed 211 patients who underwent surgical treatment of pancreatic tumors from January 2013 to January 2019 at the Sun Yat-sen Memorial Hospital of Sun Yat-sen University, Guangzhou, China. All patients had no pre-treatment, achieved $\mathrm{R} 0$ resection, and had no severe surgical complications after surgery. In all patients, adenocarcinoma was confirmed by a postoperative histological examination, and other histological variants were excluded, such as mucinous cystic adenocarcinoma, intraductal papillary adenocarcinoma, acinar cell carcinoma, and endocrine carcinoma. Patients with borderline resectable pancreatic cancer, distally metastatic pancreatic cancer, and R1 resected pancreatic cancer were excluded. All patients underwent regular postoperative adjuvant chemotherapy (based on S1 or gemcitabine). The study was conducted in accordance with the Declaration of Helsinki (as revised in 2013). The study was approved by institutional ethics committee of Sun Yat-sen Memorial Hospital (NO. SYSEC-KY-KS-2021-010) and individual consent for this retrospective analysis was waived.

\section{Analysis of high-risk factors}

This study investigated the relationship between abdominal and/or back pain, CA19-9, tumor pathological features, and prognosis, and the relationship between pain and possible causes. The variables indicated by previous investigations were classified as laboratory tests, pathological findings, and CT images. The laboratory tests investigated were as follows: tumor markers such as carbohydrate antigen 19-9 (CA19-9), c-reactive protein (CRP), carbohydrate antigen 125 (CA125), carcinoembryonic antigen(CEA), $\alpha$-fetoprotein(AFP), serum amylase (AMY), neutrophilto-lymphocyte ratio (NLR), platelet-to-lymphocyte ratio (PLR), lymphocyte-to-monocyte ratio (LMR), and total bilirubin (TBil). The pathological factors investigated were as follows: location of the pancreatic tumor, pancreatic tumor size, degree of tumor differentiation and tumor invasion of intra- or extra pancreatic tissues such as invasion of the anterior pancreatic capsule (IPC), pancreatic perineural invasion (PNI), and lymph node metastasis (LNM). The CT/MRI images identified the location of the pancreatic tumor, pancreatic tumor size, and pancreatic duct diameter.

The cut-off values in our data were determined based on previous reports and quartiles of preoperative CA19-9 levels (8-10): $<35, \geq 35, \geq 500$, and $\geq 1,000 \mathrm{U} / \mathrm{mL}$, meanwhile ROC analysis was also used to calculate the vales, to find the best one. The levels of abdominal and/or back pain were determined by the visual analogue scale (VAS) and were divided into three groups (11): none (0), mild [1-3], moderate [4-6], and severe [7-10]. Tumor size classification 
Table 1 Summary of patient characteristics before surgery

\begin{tabular}{|c|c|}
\hline Characteristic & Patients $(\mathrm{N}=211)$ [\%] \\
\hline \multicolumn{2}{|l|}{ Sex } \\
\hline Male & $112[53]$ \\
\hline Female & $99[47]$ \\
\hline Age, median [range], y & 63 [26-81] \\
\hline \multicolumn{2}{|l|}{ CA19-9 level, U/mL } \\
\hline Median (range) & $202.5(0-15,968)$ \\
\hline$<35$ (normal) & 39 [20] \\
\hline$\geq 35$ (elevated) & $156[80]$ \\
\hline \multicolumn{2}{|l|}{ Tumor site } \\
\hline Head & $162[77]$ \\
\hline Body & $40[19]$ \\
\hline Tail & 9 [4] \\
\hline \multicolumn{2}{|l|}{ Surgery } \\
\hline Pancreaticoduodenectomy & $168[80]$ \\
\hline Distal pancreatectomy & $43[20]$ \\
\hline \multicolumn{2}{|l|}{ Abdominal and/or back pain } \\
\hline None (VAS =0) & $44[21]$ \\
\hline Mild (VAS =1-3) & $76[36]$ \\
\hline Moderate (VAS =4-6) & $84[40]$ \\
\hline Severe (VAS =7-10) & 7 [3] \\
\hline
\end{tabular}

CA19-9, cancer antigen 19-9; VAS, visual analogue scale.

was based on the latest NCCN guidelines (12): $<2, \geq 2$, and $\geq 4 \mathrm{~cm}$.

\section{Statistical methods}

The Mann-Whitney $U$ test for quantitative variables or the $\chi^{2}$ test for qualitative variables were used to examine the associations between pain and each clinicopathological variable. The relationship between abdominal and/or back pain intensity and each variable was assessed using Spearman's rank correlation test or $\chi^{2}$ test. Optimal cutoff values of the continuous NLR, PLR, and LMR were calculated by applying receiver operating curve (ROC) analysis. Independent risk factors were assessed using multiple logistic regression analysis. Survival curves were calculated using the Kaplan-Meier method and differences in survival were evaluated by log-rank tests. Survival time was calculated from the date of surgery. All $\mathrm{P}$ values were two tailed, and $\mathrm{P}$ values $<0.05$ were considered significant.

\section{Results}

A summary of patient characteristics before surgery is listed in Table 1. The main surgical procedures performed were pancreaticoduodenectomy in 168 (80\%) patients and distal pancreatectomy in $43(20 \%)$ patients. Abdominal and/or back pain was divided into four levels: none, mild, moderate, and severe. Patients with moderate pain needed NSAIDs to control pain and were effective. Only 7 patients ("extreme pain") complained of severe pain and needed NSAIDs combined with opioid analgesics, and their tumors were all located at the head of the pancreas.

\section{Relationship between OS and high-risk features}

LNM, NLR, and PLR were significant variables related to OS. An investigation of the relationship between CA199 and OS showed that only CA19-9 $\geq 1,000 \mathrm{U} / \mathrm{mL}$ was statistically significant. There was no significant relationship between pain and OS, and there was no statistically significant relationship between pancreatic cancer tumor size and OS according to NCCN classification. However, we performed a more detailed classification of pancreatic cancer tumor size and found that tumors greater than $6 \mathrm{~cm}$ were significantly associated with OS (Figure 1).

TBil was subjected to ROC analysis according to CA199 , and the best cut-off value was $17.25 \mu \mathrm{mol} / \mathrm{L}$. An analysis of the relationship between CA19-9 and OS in patients with lower than the cut-off value of TBil found that CA19$9 \geq 500 \mathrm{U} / \mathrm{mL}$ was statistically associated with prognosis (Figure 2).

\section{Factors related to abdominal and/or back pain}

The location of the pancreatic tumor, AMY, serum lipase, pancreatic duct dilatation (PDD), NLR, and PLR were significant variables related to abdominal and/or back pain. According to the diagnostic criteria for chronic pancreatitis (13), chronic pancreatitis caused by pancreatic tumors was statistically related to pain. Other laboratory tests (CRP, CA125, CEA, AFP) were not significantly correlated with pain, as were other pathological factors, such as differentiation of tumors, pancreatic tumor size, PNI, and LNM (Table 2). The relationship between pain and OS was studied according to the location of the tumor. 
A

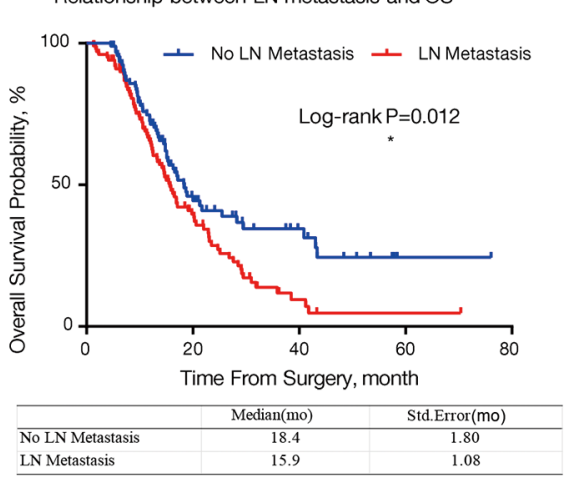

B

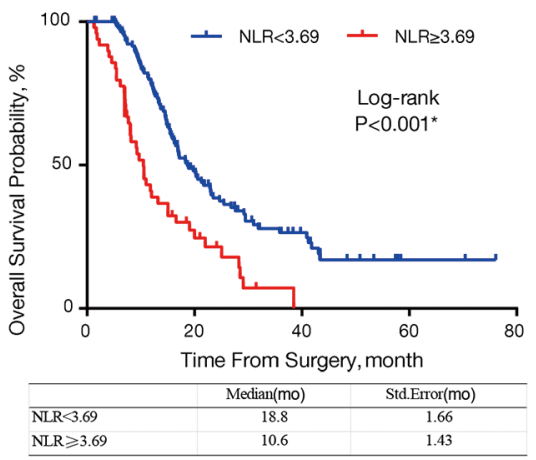

C Relationship between PLR and OS
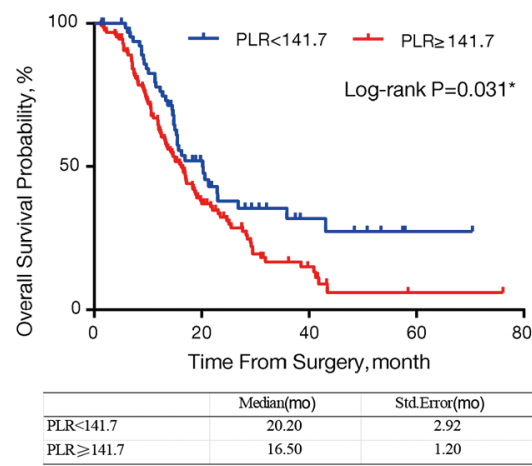

D

Relationship between CA19-9 and OS
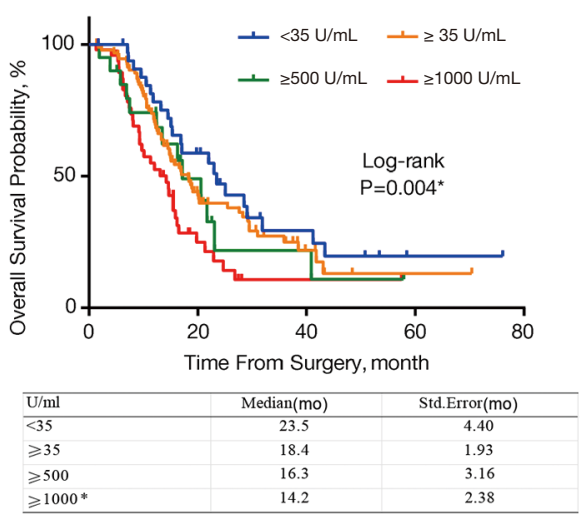

$\mathrm{F}$

Relationship between tumor size and OS -1

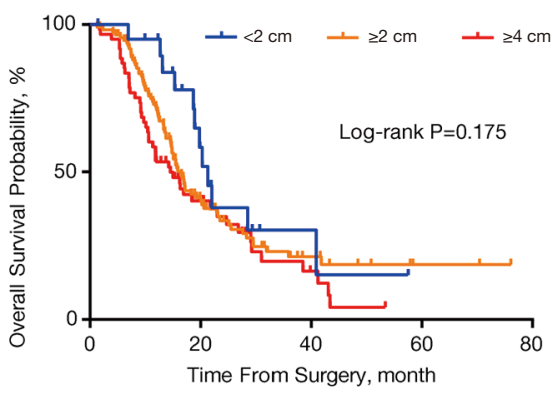

$\mathrm{E}$

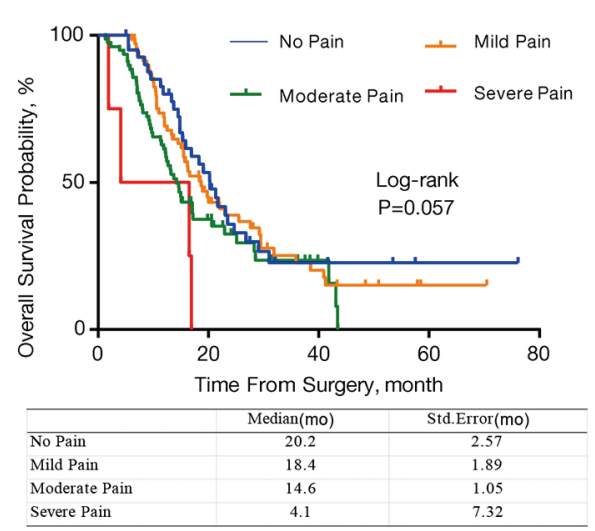

G

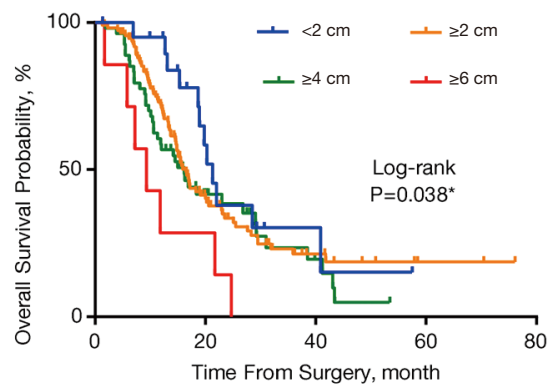

\begin{tabular}{l|c|c}
\hline & Median(mo) & Std.Error(mo) \\
\hline$<2 \mathrm{~cm}$ & 21.3 & 1.33 \\
\hline $2 \mathrm{~cm}$ & 16.6 & 0.97 \\
$\geqslant 4 \mathrm{~cm}$ & 16.2 & 2.68 \\
$\geqslant 6 \mathrm{~cm}{ }^{*}$ & 9.3 & 2.75 \\
\hline
\end{tabular}

Figure 1 The relationship between overall survival (OS) and high-risk features, such as (A) lymph node (LN) metastasis, (B) neutrophil-tolymphocyte ratio (NLR), (C) platelet-to-lymphocyte ratio (PLR), (D) cancer antigen 19-9 (CA19-9), (E) abdominal and/or back pain, and tumor size (F,G). Through receiver operating characteristic (ROC) analysis, the best cut-off points for NLR and PLR were 3.96 [area under the ROC curve (AUC) $0.589, \mathrm{P}=0.032^{*}$ ] and 141.7 (AUC $0.583, \mathrm{P}=0.47^{*}$ ), respectively. *, $\mathrm{P}<0.05$ was statistically significant. 


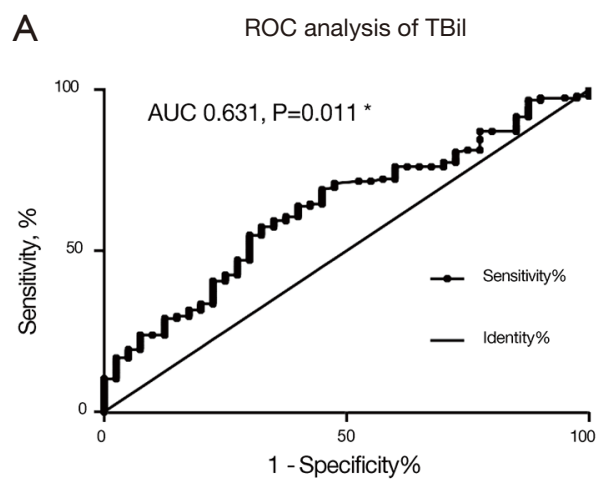
B Relationship between CA19-9 and OS in patients
with TBil $<17.25 \mu \mathrm{mol} / \mathrm{L}$

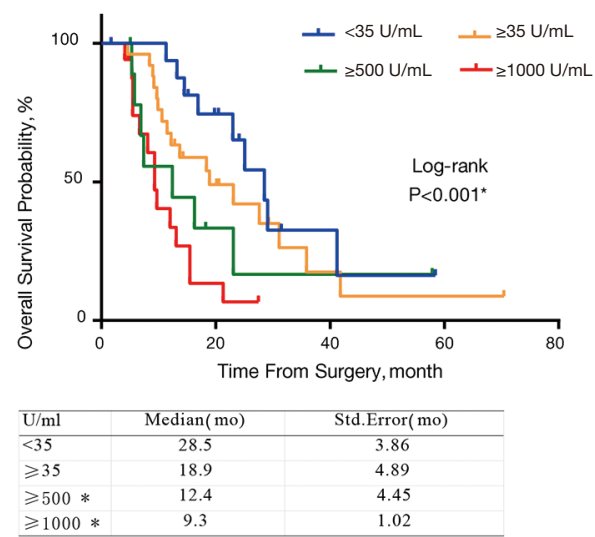

Figure 2 The relationship between CA19-9 and OS in patients with lower than cut-off value of TBil. (A) Receiver operating characteristic (ROC) analysis of total bilirubin (TBil) according to CA19-9, and the best cut-off value was $17.25 \mu \mathrm{mol} / \mathrm{L}$; (B) the relationship between CA19-9 and overall survival (OS) in patients with TBil $<17.25 \mu \mathrm{mol} / \mathrm{L} .{ }^{*}, \mathrm{P}<0.05$ was statistically significant.

It was found that there was no statistical correlation between pain and OS in pancreatic head tumors, but there was a statistical correlation between moderate pain and OS in pancreatic tail tumors (Figure 3).

\section{CA19-9 cut-off point and other bigh-risk features}

CA19-9 was calculated by ROC analysis. The best cut-off value for CA19-9 was found to be $340.7 \mathrm{U} / \mathrm{mL}$. In patients with higher CA19-9, it found that pain was significantly associated with OS, and mild, moderate, and severe pain had a worse prognosis. Pain was also significantly associated with PNI. We then used $500 \mathrm{U} / \mathrm{mL}$ as the cut-off point for CA19-9 which showed similar results (Figure 4). There was no statistical association between CA19-9 and tumor size after CA19-9 cut-off classification.

\section{Tumor size cut-off point and abdominal and/or back pain}

After tumor size was classified according to $2 \mathrm{~cm}$, in patients with pancreatic cancer greater than $2 \mathrm{~cm}$, the relationship between pain intensity and prognosis was similar to that of the unclassified patients. When tumor size was classified according to $4 \mathrm{~cm}$, patients with moderate and severe pain were found to have worse prognosis (Figure 4).

\section{Discussion}

In this study, we only studied patients with clearly resectable pancreatic cancer, and identified the specific values of high-risk features. We found that CA19-9, tumor size, and abdominal and/or back pain for the prediction of prognosis was different from previous studies.

CA19-9, a sialylated Lewis A blood group antigen, is the best-validated and most clinically useful biomarker for the early detection and surveillance of pancreatic cancer. In the past, studies of patients with resectable pancreatic cancer identified a cut-off point of $200 \mathrm{U} / \mathrm{mL}$ for preoperative CA19-9, and patients with more than $200 \mathrm{U} / \mathrm{mL}$ had a significantly worse prognosis than those with less than $200 \mathrm{U} / \mathrm{mL}$ (8). Comparing different CA199 levels, patients with CA19-9 greater than $500 \mathrm{U} / \mathrm{mL}$ had a worse prognosis (10). However, these studies were based on clearly resectable pancreatic cancer and borderline resectable pancreatic cancer, including postoperative pathology confirmed $\mathrm{R} 0$ resection and $\mathrm{R} 1$ resection. The scope of the influence of CA19-9 on prognosis has expanded. Neoadjuvant chemotherapy has confirmed the advantages of borderline resectable pancreatic cancer in many studies, though our study did not involve borderline tumors. For clearly resectable pancreatic cancer with R0 resection, we obtained a different range of CA19-9, as the best cut-off point for CA19-9 was $340.7 \mathrm{U} / \mathrm{mL}$, and patients with a CA19-9 greater than $1000 \mathrm{U} / \mathrm{mL}$ showed significant differences in prognosis. Therefore, "markedly" elevated CA19-9 may be defined as higher than 1,000 U/mL in these patients. CA19-9 may be elevated as a result of biliary infection, inflammation, or obstruction $(14,15)$. 
Table 2 The characteristics of patients with clearly resectable pancreatic cancer and pain

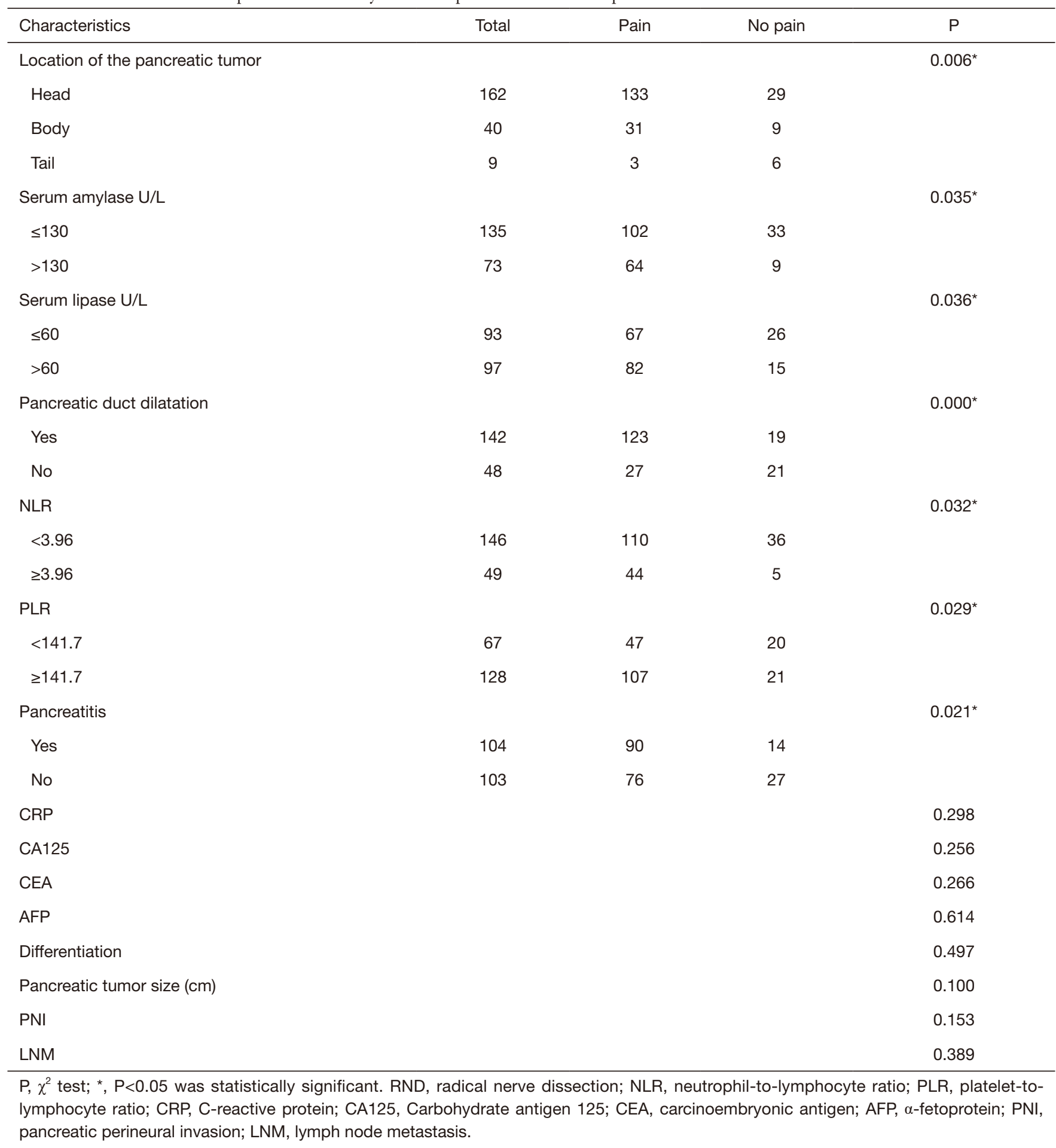

Therefore, we also reviewed the TBil data of these patients and conducted a correlation study with CA19-9. It was found that, through ROC analysis, the cut-off value of
TBil was $17.25 \mu \mathrm{mol} / \mathrm{L}$, which was equal to the standard value of TBil $(17.1 \mu \mathrm{mol} / \mathrm{L})$. To analyze the relationship between CA19-9 and OS in patients with normal bilirubin, 

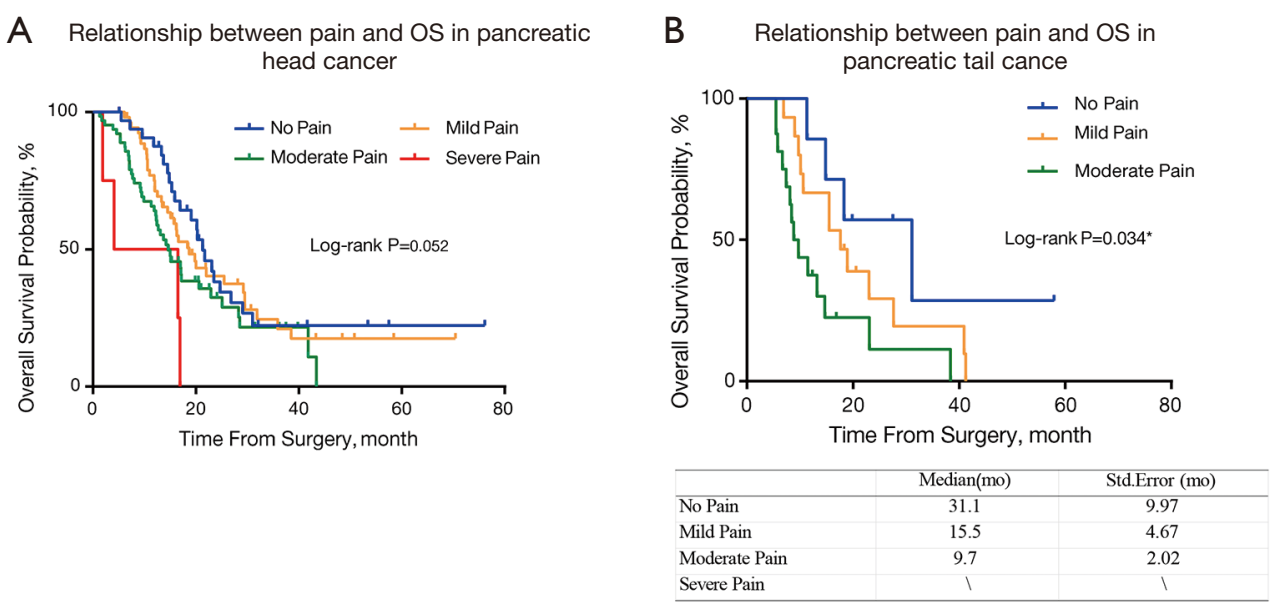

Figure 3 The relationship between pain and OS was studied according to the location of the tumor. (A) No statistical correlation was found between pain and overall survival (OS) for pancreatic head tumors; (B) the relationship between pain and OS for pancreatic tail tumors. *, $\mathrm{P}<0.05$ was statistically significant.

it was found that the prognosis of patients with CA19-9 $\geq 500 \mathrm{U} / \mathrm{mL}$ was significantly different. Therefore, we believe that in patients with clearly resectable pancreatic cancer, CA19-9 >500 U/mL with normal TBil and CA19-9 $>1,000 \mathrm{U} / \mathrm{mL}$ with elevated TBil had significantly worse prognosis.

The NCCN pancreatic cancer guidelines before 2018 classified tumor size by $2 \mathrm{~cm}$ according the 7 th edition [2010] of the American Joint Committee on Cancer (AJCC) Cancer Staging Manual (16), and the new version of the pancreatic cancer guidelines in 2018 classified the size of pancreatic cancer into 3 levels according to the 8 th edition [2017] of the AJCC Cancer Staging Manual (12). Clinicians pay more attention to the impact of tumor size on prognosis. Our study of patients with clearly resectable pancreatic cancer found that there was no statistical difference in the size of the tumors when classified into three levels. However, after dividing into four groups, the tumors with a diameter greater than $6 \mathrm{~cm}$ showed a significantly worse prognosis. Therefore, "large" primary tumors may be defined as larger than $6 \mathrm{~cm}$ in clearly resectable pancreatic cancer patients.

Current studies of pancreatic cancer have demonstrated that pain is associated with prognosis, and more severe pain predicts a worse prognosis $(7,17,18)$. These studies include advanced unresectable pancreatic cancer and borderline resectable pancreatic cancer. Abdominal and/or back pain studies for resectable pancreatic cancer also include borderline resectable pancreatic cancer and postoperative R1 resected tumor patients $(7,19)$. Our study found that pain was not significantly associated with prognosis, although patients with severe pain had significantly shorter OS, but only 7 patients had severe pain, and "extreme pain" patients with resectable disease were rare in clinical practice. There are two main causes of abdominal and/or back pain caused by pancreatic cancer: chronic pancreatitis caused by pancreatic cancer and tumor invasion of nerves (20). We studied pain in clearly resectable pancreatic cancer patients and found that there was a significant correlation between pain and pancreatitis, which was also significantly associated with tumor sites, and significantly associated with systemic inflammation, but were not associated with PNI. Based on our findings, we believe that pain in early pancreatic cancer cannot be used alone as a prognostic indicator for pancreatic cancer. Patients were divided into two groups according to the best cut-off point of CA19-9 (340.7 U/mL). In the group of patients with CA19-9 greater than $340.7 \mathrm{U} / \mathrm{mL}$, we found that pain was significantly associated with prognosis, and patients with mild, moderate, and severe pain had a worse prognosis. At the same time, pain was significantly related to PNI, suggesting that the possible cause of pain was invasion of nerves. Similarly, when tumor size and pain were combined, it was found that when the cut-off value of tumor size was $2 \mathrm{~cm}$, the relationship between pain and OS was similar to uncut-off tumor size. However, with $4 \mathrm{~cm}$ as the cut-off point, moderate and 
A

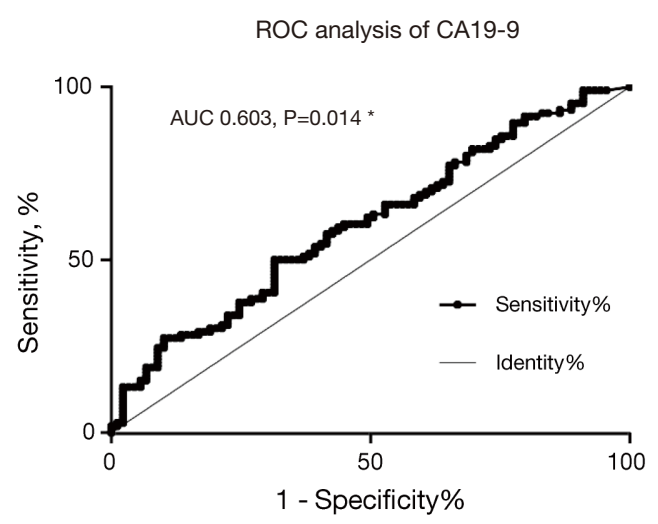

C

Relationship between pain and PNI

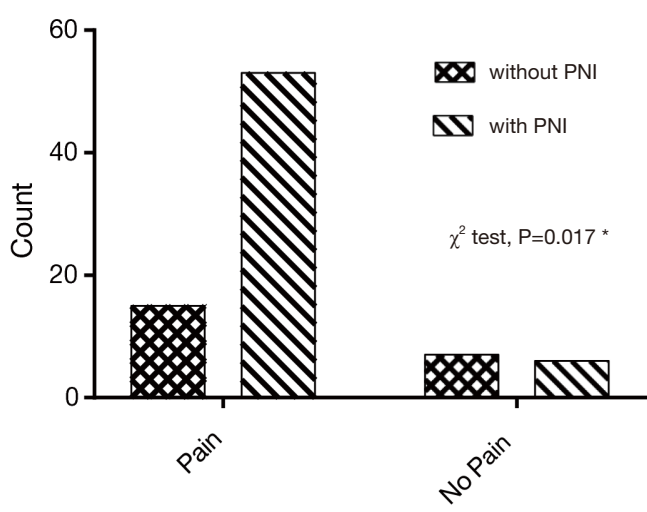

B

Relationship between pain and OS in patients with CA19-9 $\geq 340.7 \mathrm{U} / \mathrm{mL}$

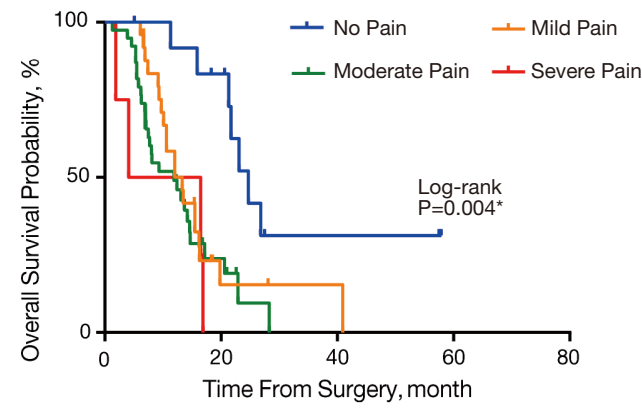

\begin{tabular}{lcc}
\hline & Median(mo) & Std.Error(mo) \\
\hline No Pain & 24.7 & 2.31 \\
Mild Pain * & 13.3 & 1.77 \\
Moderate Pain * & 11.9 & 2.89 \\
Severe Pain * & 4.1 & 7.32 \\
\hline
\end{tabular}

D

Relationship between pain and OS with tumor size $\geq 4 \mathrm{~cm}$

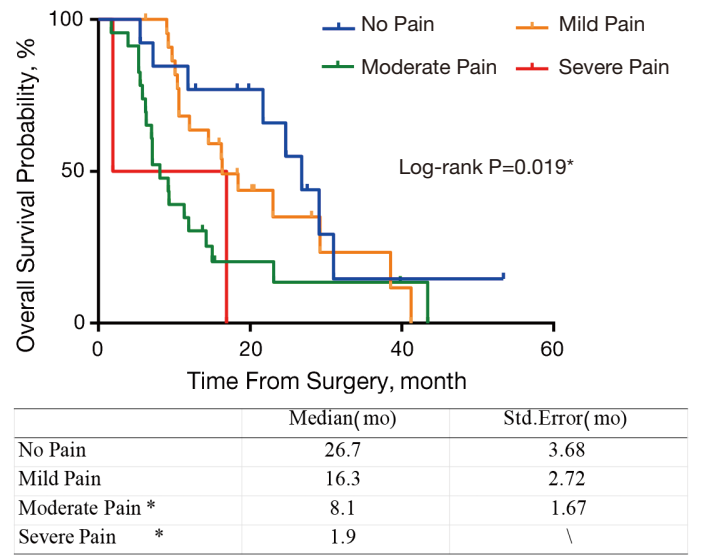

Figure 4 The relationship between pain and OS with the cut-off point of CA19-9/tumor size. (A) Receiver operating characteristic (ROC) analysis of CA19-9, and the best cut-off value was $340.7 \mathrm{U} / \mathrm{mL}$; (B) the relationship between pain and overall survival (OS) in patients with CA19-9 $\geq 340.7 \mathrm{U} / \mathrm{mL}$; (C) the relationship between pain and pancreatic perineural invasion (PNI) in patients with CA19-9 $\geq 340.7 \mathrm{U} / \mathrm{mL}$; (D) relationship between pain and overall survival (OS) with tumor size $\geq 4 \mathrm{~cm}$. ${ }^{*}, \mathrm{P}<0.05$ was statistically significant.

severe pain showed a significantly worse prognosis.

In our study, although LNM of pancreatic cancer was found to be associated with prognosis, LNM was confirmed by postoperative pathology. It is difficult to determine whether lymph node enlargement based on imaging examinations before surgery was caused by inflammation or tumor metastasis, and whether resolution of lymph nodes before surgery was also due to the influence of imaging inspection instruments. It is therefore difficult to get a reliable conclusion by retrospective studies, and further studies involving the radiological department should be performed in the future.

Through this study, we found that the specific values of high-risk features for clearly resectable pancreatic cancer (R0 resection) were: (I) patients with CA19-9 $\geq 1,000 \mathrm{U} / \mathrm{mL}$; (II) patients with CA19-9 $\geq 500 \mathrm{U} / \mathrm{mL}$ combined with normal TBil; (III) primary tumor greater than $6 \mathrm{~cm}$; (IV) 10 times elevated CA19-9 levels with abdominal/back pain; (V) primary tumor greater than $4 \mathrm{~cm}$ combined with moderate or severe pain. "Extreme pain" can be classified 
as severe pain (VAS 7-10). Patients with high-risk features may consider receiving neoadjuvant chemotherapy before surgery, and the effect of neoadjuvant chemotherapy may require further research in the future.

\section{Conclusions}

We have studied and clarified the high-risk features for clearly resectable pancreatic cancer with $\mathrm{R} 0$ resection, and found that in these pancreatic cancer patients, abdominal and/or back pain may not be used as a prognostic indicator alone, though in combination with CA19-9 or tumor size it may be more valuable for predicting prognosis.

\section{Acknowledgments}

Funding: Guangdong Medical Science and Technology Research Fund, A2020240.

\section{Footnote}

Reporting Checklist: The authors have completed the STROBE reporting checklist. Available at http://dx.doi. org/10.21037/gs-20-877

Data Sharing Statement: Available at http://dx.doi. org/10.21037/gs-20-877

Conflicts of Interest: All authors have completed the ICMJE uniform disclosure form (available at http://dx.doi. org/10.21037/gs-20-877). The authors have no conflicts of interest to declare.

Ethical Statement: The authors are accountable for all aspects of the work in ensuring that questions related to the accuracy or integrity of any part of the work are appropriately investigated and resolved. The study was conducted in accordance with the Declaration of Helsinki (as revised in 2013). The study was approved by institutional ethics committee of Sun Yat-sen Memorial Hospital (No.: SYSEC-KY-KS-2021-010) and individual consent for this retrospective analysis was waived.

Open Access Statement: This is an Open Access article distributed in accordance with the Creative Commons Attribution-NonCommercial-NoDerivs 4.0 International License (CC BY-NC-ND 4.0), which permits the noncommercial replication and distribution of the article with the strict proviso that no changes or edits are made and the original work is properly cited (including links to both the formal publication through the relevant DOI and the license). See: https://creativecommons.org/licenses/by-nc-nd/4.0/.

\section{References}

1. Tempero MA, Malafa MP, Al-Hawary M, et al. NCCN Clinical Practice Guidelines in Oncology: Pancreatic Adenocarcinoma (Version 1.2019). J Natl Compr Canc Netw 2019;17:202-10.

2. Oettle H, Neuhaus P, Hochhaus A, et al. Adjuvant chemotherapy with gemcitabine and long-term outcomes among patients with resected pancreatic cancer: the CONKO-001 randomized trial. JAMA 2013;310:1473-81.

3. Neoptolemos JP, Stocken DD, Bassi C, et al. Adjuvant chemotherapy with fluorouracil plus folinic acid vs gemcitabine following pancreatic cancer resection: a randomized controlled trial. JAMA 2010;304:1073-81.

4. Kalser MH, Ellenberg SS. Pancreatic cancer. Adjuvant combined radiation and chemotherapy following curative resection. Arch Surg 1985;120:899-903.

5. Massucco P, Capussotti L, Magnino A, et al. Pancreatic resections after chemoradiotherapy for locally advanced ductal adenocarcinoma: analysis of perioperative outcome and survival. Ann Surg Oncol 2006;13:1201-8.

6. Quiros RM, Brown KM, Hoffman JP. Neoadjuvant therapy in pancreatic cancer. Cancer Invest 2007;25:267-73.

7. Okusaka T, Okada S, Ueno H, et al. Abdominal pain in patients with resectable pancreatic cancer with reference to clinicopathologic findings. Pancreas 2001;22:279-84.

8. Ferrone CR, Finkelstein DM, Thayer SP, et al. Perioperative CA19-9 Levels Can Predict Stage and Survival in Patients With Resectable Pancreatic Adenocarcinoma. J Clin Oncol2006;24:2897-902.

9. Waraya M, Yamashita K, Katagiri H, et al. Preoperative Serum CA19-9 and Dissected Peripancreatic Tissue Margin as Determiners of Long-Term Survival in Pancreatic Cancer. Ann Surg Oncol 2009;16:1231-40.

10. Kondo N, Murakami Y, Uemura K, et al. Prognostic Impact of Perioperative Serum CA 19-9 Levels in Patients with Resectable Pancreatic Cancer. Ann Surg Oncol 2010;17:2321-9.

11. Hirschfeld G, Zernikow B. Cut points for mild, moderate, and severe pain on the VAS for children and adolescents: what can be learned from 10 million ANOVAs? Pain 2013;154:2626-32. 
12. Amin MB, Edge S, Greene F, et al. The Eighth Edition AJCC Cancer Staging Manual: Continuing to build a bridge from a population-based to a more "personalized" approach to cancer staging. CA Cancer J Clin 2017;67:93-9.

13. Whitcomb DC, Shimosegawa T, Chari ST, et al. International consensus statements on early chronic Pancreatitis. Recommendations from the working group for the international consensus guidelines for chronic pancreatitis in collaboration with The International Association of Pancreatology, American Pancreatic Association, Japan Pancreas Society, PancreasFest Working Group and European Pancreatic Club. Pancreatology 2018;18:516-27.

14. Mann DV, Edwards R, Ho S, et al. Elevated tumour marker CA19-9: clinical interpretation and influence of obstructive jaundice. Eur J Surg Oncol 2000;26:474-9.

15. Marrelli D, Caruso S, Pedrazzani C, et al.CA19-9 serum levels in obstructive jaundice: clinical value in benign and malignant conditions. Am J Surg 2009;198:333-9.

16. Edge S, Byrd DR, Compton CC, et al.The American Joint

Cite this article as: $\mathrm{Xu} \mathrm{D}$, Wang J, Liu T, Huang Z, Luo J, Chen Y, Lu Y. Quantitative definitions of pain, CA19-9, and tumor size as high-risk features of resectable pancreatic cancer: a single-center retrospective cohort study. Gland Surg 2021;10(2):770-779. doi: 10.21037/gs-20-877
Committee on Cancer: the 7th edition of the AJCC cancer staging manual and the future of TNM. Ann Surg Oncol 2010;6:1471-4.

17. Koulouris AI, Banim P,Hart AR. Pain in Patients with Pancreatic Cancer: Prevalence, Mechanisms, Management and Future Developments. Digest Dis Sci 2017;62:861-70.

18. Wyse JM, Carone M, Paquin SC, et al. Randomized, double-blind, controlled trial of early endoscopic ultrasound-guided celiac plexus neurolysis to prevent pain progression in patients with newly diagnosed, painful, inoperable pancreatic cancer. J Clin Oncol 2011;29:3541-6.

19. Kelsen DP, Portenoy R, Thaler H, et al. Pain as a predictor of outcome in patients with operable pancreatic carcinoma. Surgery 1997;122:53-9.

20. Bapat AA, Hostetter G, Von Hoff DD et al. Perineural invasion and associated pain in pancreatic cancer. Nat Rev Cancer 2011;11:695-707.

(English Language Editor: C. Betlazar-Maseh) 